$\$$

\title{
A Novel Energy Efficient Resource Management System in Cloud Computing Environment.
}

\author{
S. K. Sonkar ${ }^{1 *}$, M.U.Kharat ${ }^{2}$ \\ ${ }^{1}$ Research Scholar, Dept. of computer engineering, KKWagh IEE\&R Nashik, SP Pune University, India. \\ ${ }^{2}$ Professor, Dept. of computer engineering METIOE Nashik,SP Pune University, India. \\ *Corresponding author E-mail: sonkar83@gmail.com
}

\begin{abstract}
Primary target of cloud provider is to provide the maximum resource utilization and increase the revenue by reducing energy consumption and operative cost. In the service providers point of view, resource allocation, resource sharing, migration of resources on demand, memory management, storage management, load balancing, energy efficient resource usage, computational complexity handling in virtualization are some of the major tasks that has to be dealt with. The major issue focused in this paper is to reduce the energy consumption problem and management of computation capacity utilization. For the same, an energy efficient resource management method is proposed to grip the resource scheduling and to minimize the energy utilized by the cloud datacenters for the computational work. Here a novel resource allocation mechanism is proposed, based on the optimization techniques. Also a novel dynamic virtual machine (VM) allocation method is suggested to help dynamic virtual machine allocation and job rescheduling to improve the consolidation of resources to execute the jobs. Experimental results indicated that proposed strategy outperforms as compared to the existing systems.
\end{abstract}

Keywords: Cloud Provider: VM Allocation; Resource Allocation; Resource utilization; Energy consumption.

\section{Introduction}

As in cloud computing, pay-per-use services are used which avoids the huge capital investment of deploying the hardware, software and other resources at home. It has some critical issues of being over-charged, privacy leak and intervention of unauthorized service providers [1-3]. In the service providers point of view, resource allocation, resource sharing, migration of resources on demand, memory management, storage management, load balancing, energy efficient resource usage, computational complexity handling in virtualization are some of the major tasks that has to be dealt with [4-8]. Resource allocation refers to the amount of resources and the types of resources that are allocated to compute the user's job. The service providers are intended to schedule and provide the resources to the users based on their needs [9-14]. The major issue focused in this research work is to reduce the energy consumption problem and management of computation capacity utilization [15-16]. It has also been one of the burning topics in the recent years of research. To overcome the above mention issues, a novel energy efficient resource management system is proposed to handle the resource scheduling and for the minimization of the energy utilized by the cloud data centers for the computational work.

The rest of the paper is organized as follows: section II presents the related work. The Contributions are mentioned in section III. Section IV represents proposed system architecture along with algorithms to efficiently handle the system. Section V describes experimental results and discussion \& section VI represents the conclusion.

\section{Related Work}

This section presents different resource allocation and scheduling techniques which helps to present our proposed work. Mehiar Dabbagh et.al [17] proposed a system for overcommitted clouds which is an energy-efficient resource allocation system. System saves enormous energy by minimizing Physical Machine (PM) overload occurrences through monitoring and prediction of VM resource usage and reducing the number of active PMs via efficient VM migration and placement tech. Fei Taoa et.al [18] addressed the optimal scheduling of computing resources (OSCR) problem consisting of energy consumption issue \& proposed the energy consumption framework and simplified it to adapt network whose load information is unavailable. Meanwhile, imbalanced load distribution is considered to represent risk on the makespan and used as an effective strategy for both to shorten the makespan and to realize load balance. Tarandeep Kaur [19] proposed Green Cloud Scheduling Model (GCSM). GCSM which is consisting of a scheduler component named as Green Cloud Scheduler for task allocation and scheduling decisions by considering the energy aware capability of heterogeneous Cloud nodes. Jieun Choi et.al [20] proposed job scheduling optimization technique which focuses on the performance \& QoS metrics in cluster and cloud environments. As a result, this technique controls the ratio of scheduling distribution for distributed environment with respect to characteristics of application and current workload.

A.V.Karthick et.al [21] proposed Multi Queue Scheduling (MQS) system which gives additional significance to choose task dynamically to accomplish the optimum cloud scheduling problem and therefore it usages unused free space in an economic manner. Vahid Arabnejad et.al [22] presented a new deadline constrained 
scheduling method, called Deadline Constrained Critical Path (DCCP) which achieves the scheduling of workloads on dynamically provisioned by cloud resources. The DCCP works in two stages. First is task prioritization and second is task assignment. This method is based on concept of Constrained Critical Paths to execute a set of tasks on the same instance in order to achieve the goal of decreasing data movement between instances. Zhiming Wang et al. [23] proposed a Particle Swarm Optimization Tabu search Mechanism, aims to improve resource use and clearly taking into account both energy consumption and revenue acquisition in minimizing completion time. The multisource job scheduler is proposed by D Huang [24] to schedule jobs efficiently having multi-resource requirements such as CPU, memory and bandwidth to improve job performance and resource utilization in a virtual machine based on cloud environment. With the growing popularity of cloud computing, it is necessary to examine various methods by which energy consumption in cloud environments can be reduced. Author [25] analyzed the effects of virtual machine allocation on energy consumption using a variety of real-world policies and a realistic testing scenario.

Shahin V et.al. [26] Proposed a platform for workload prediction and VM placement in cloud computing Datacenter. First an estimation module was introduced to predict the incoming load of the DC. Then, schedulers were designed to determine the optimal assignment of VMs to the PMs.

\section{Contribution}

- A novel resource allocation mechanism is proposed based on the optimization techniques.

- A novel dynamic virtual machine allocation method is proposed, to help dynamic virtual machine allocation and job rescheduling to improve the consolidation of resources to execute the jobs.

- Additionally, a novel virtual machine migration technique is proposed based on the live migration for dynamic reallocation of resources.

\section{System Model}

Based on the research gap, here we have proposed a system to design and analyze energy efficient resource management method by allocation of various resources to virtual machines for higher utilization of resources and to reduce energy consumption. The proposed work implementation is based on the architecture shown in Fig 1.

\subsection{System Workflow}

In this work we have taken input parameters from cloudsim and applied federation-> Clustering->job scheduling ->Prioritization.

The system workflow is explained as follows:

1. First of all, create datacenters

2. In next step, create number of cloud brokers

3. Next step create number of Virtual Machines

4. Then creating the number of cloudlets or jobs

5. Next step is perform the resource scheduling

6. Then perform Resource consolidation

7. After performing Resource consolidation, Dynamic Virtual machine allocation is performed for the same following things are required -

i) Job rescheduling

ii) Workload monitoring

8. If the workload is overloaded to VM then VM migration is required to perform else the VM should be in stable state or switched off.

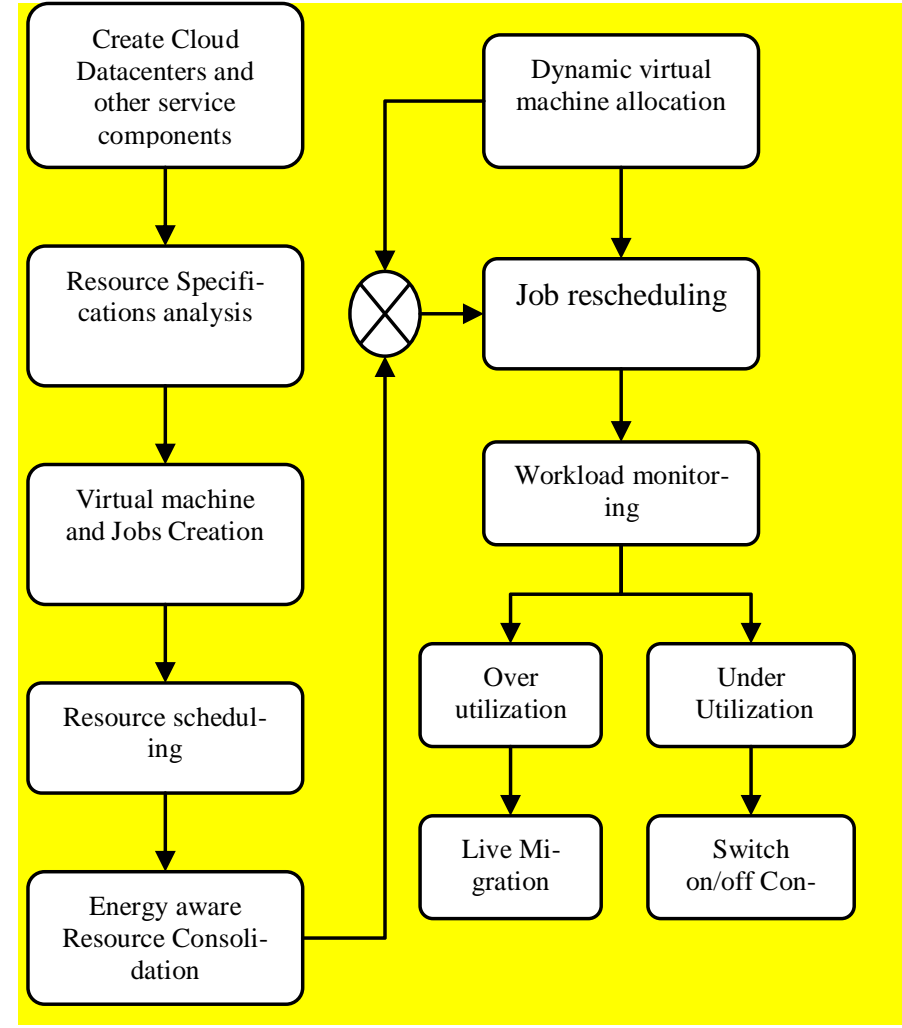

Fig.1. System Workflow

Above steps are further elaborated as follows:

\subsubsection{Datacenter Creation:}

First steps is to create a set of Datacenters. Datacenters are created with the features like RAM size, bandwidth, and number of host in each datacenter. These are the parameters of datacenters that will affect the end user when he tries to access a cloud service or a web service.

\subsubsection{Cloud Broker Creation:}

The next step is cloud broker creation, it acts a third-party mediator. And works as a connecting bridge between the user and virtual machine. Broker creation steps includes Broker name, Broker id.

\subsubsection{VMs Creation:}

The next step is VM creation. Each Datacenter consists of one or more physical machines \& each physical machine can hold more than one virtual machines. This Virtual machine are submitted to respective broker. Created VM is having parameter like VM id, Broker id, Datacenter id, CPU, Memory, and Bandwidth etc.

\subsubsection{Cloudlet or Job Creation:}

The next step is Cloudlet creation. Each cloudlets is having their unique ID and the cloudlet is submitted to respective cloud broker. The features of cloudlets are Cloudlet Length in Million Instruction per Second (MIPS), file size is measure in bytes, and types of cloudlets request for ex. CPU, memory and bandwidth.

\subsubsection{Energy Consumption:}

The next step is to calculate Energy consumption. For the same first we need to calculate Computing energy comsumption. Computing energy comsumption is calculated as follows:

Cpec $=$ Computing energy comsumption

Ep $\leftarrow$ MIPS of one Processing Element $(\mathrm{PE}) /$ time

$\mathrm{Tp} \leftarrow$ MIPS of cloudlet 
Din $\leftarrow$ cloudlet size

$\mathrm{Cpec}=\mathrm{Ep}+\mathrm{T} p+$ Din

Then second step is Communication energy consumption is calculated as follows:

Cmec $\leftarrow$ Communication energy comsumption

Dc $\leftarrow$ bandwidth

Din $\leftarrow$ cloudlet size

Ec $\leftarrow$ Dc/time

$\mathrm{Cmec}+=(\mathrm{Din} /(\mathrm{Dc} / \mathrm{Ec}))$

Then summation of computing energy comsumption and communication energy consumption is called energy consumption.

Energy consumption $=$ consumption energy + communication energy

(3)

Energy consumption is used to form the data centers into clusters. Fig. 2 shows the computing energy consumption.

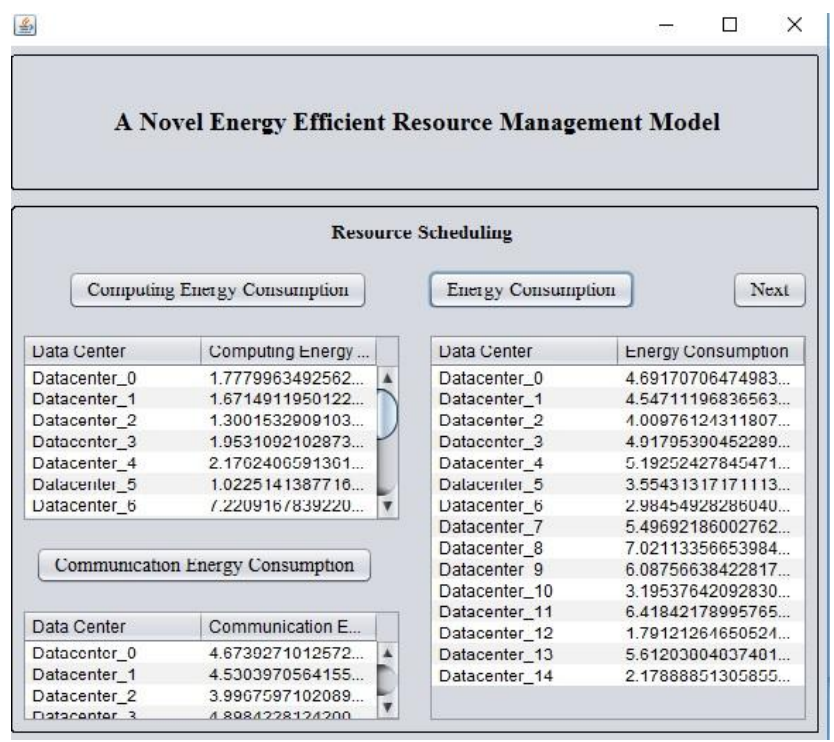

Fig.2: Computing Energy Consumption

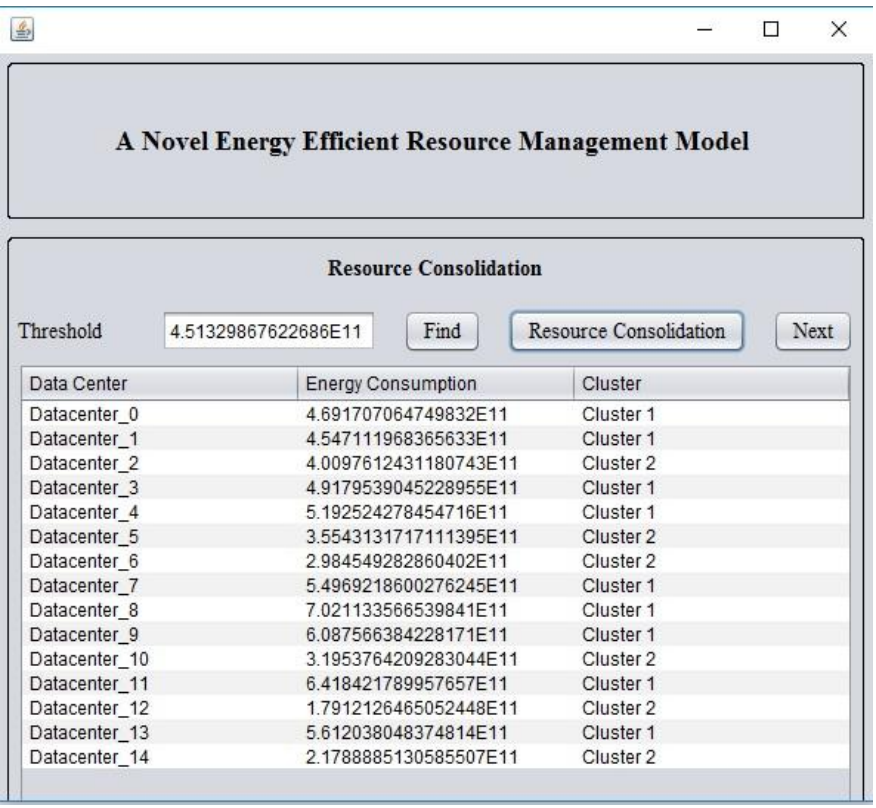

Fig.3: Resource Consolidation

Threshold is the value used to split the data centers into clusters. Threshold is calculated dynamically. Based on the energy consumption of data centers, threshold is calculated automatically.

Threshold=total energy consumption / number of data centers
If datacenters have high energy consumption than threshold means, they are formed as cluster1. Otherwise declared as cluster2, shown in fig. 3 .

If energy consumption $>$ threshold

Datacenter $=$ cluster 1

Else

Datacenter=cluster2

Workload for the datacenter is predicted for the reallocation of the cloudlets.Cloudlets in over utilized data centers are allocated to under utilized data centers. Workload is calculated as follows:

Workload $=$ Resource Capacity - Needs

If workload is greater than zero, then data center is underutilized. Otherwise data center is called as over utilized.

\subsubsection{Job Prioritization:}

Job Prioritization can be done by applying sequencing algorithm, as shown in Fig.4.In sequencing algorithm jobs with lowest execution is executed first and job with higher execution time is executed subsequently.

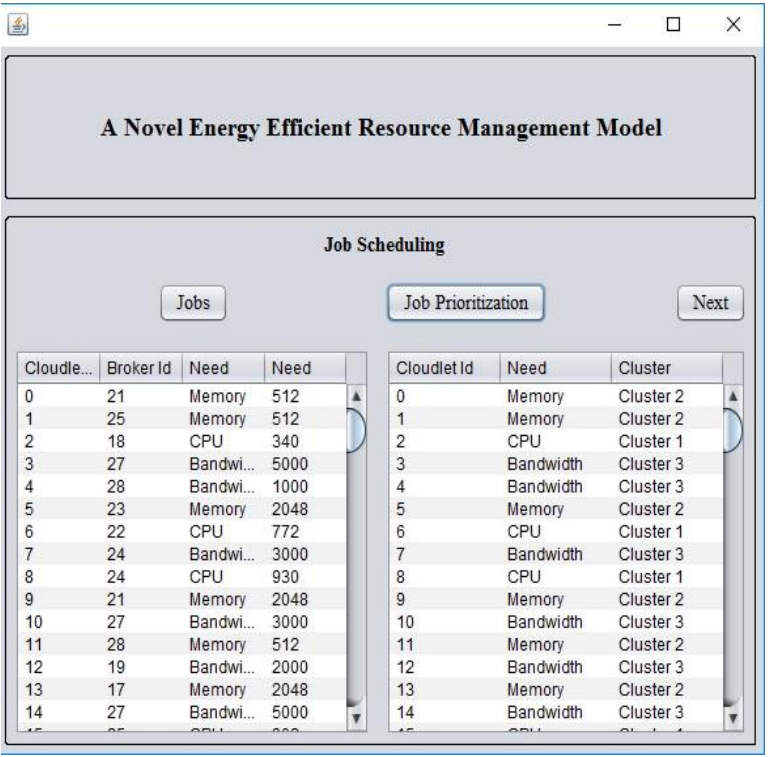

Fig.4: Job prioritization

\subsubsection{Job Allocation:}

Jobs are allocated on different virtual machines based on VM capacity and cloudlets needs, as shown in Left half of Fig.5.

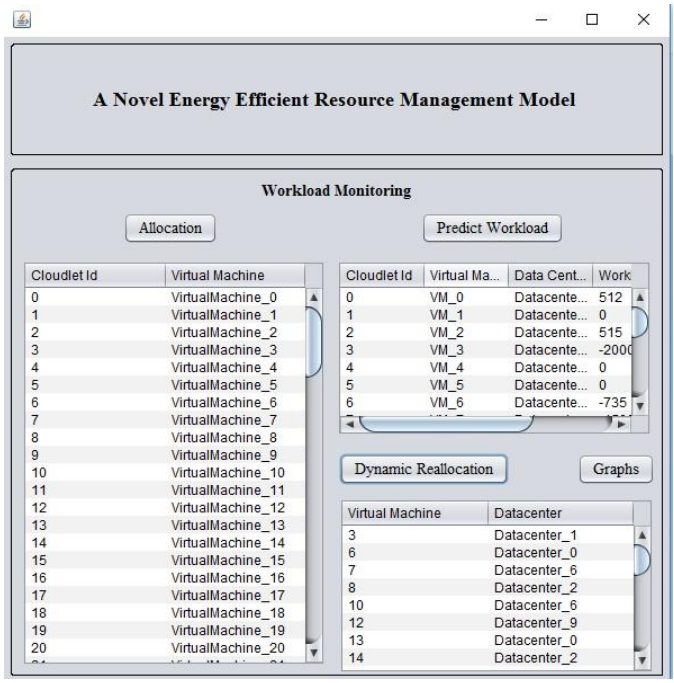

Fig.5: Workload Monitoring 


\subsubsection{Predicting Workload:}

Cloudlets are allocated to datacenters. Workload is predicted by VM Capacity - Cloudlet needs. For the VM which is over utilized, datacenters needs the migration. VM's in the over utilized datacenters are migrated to underutilized datacenters.

\subsection{Algorithm}

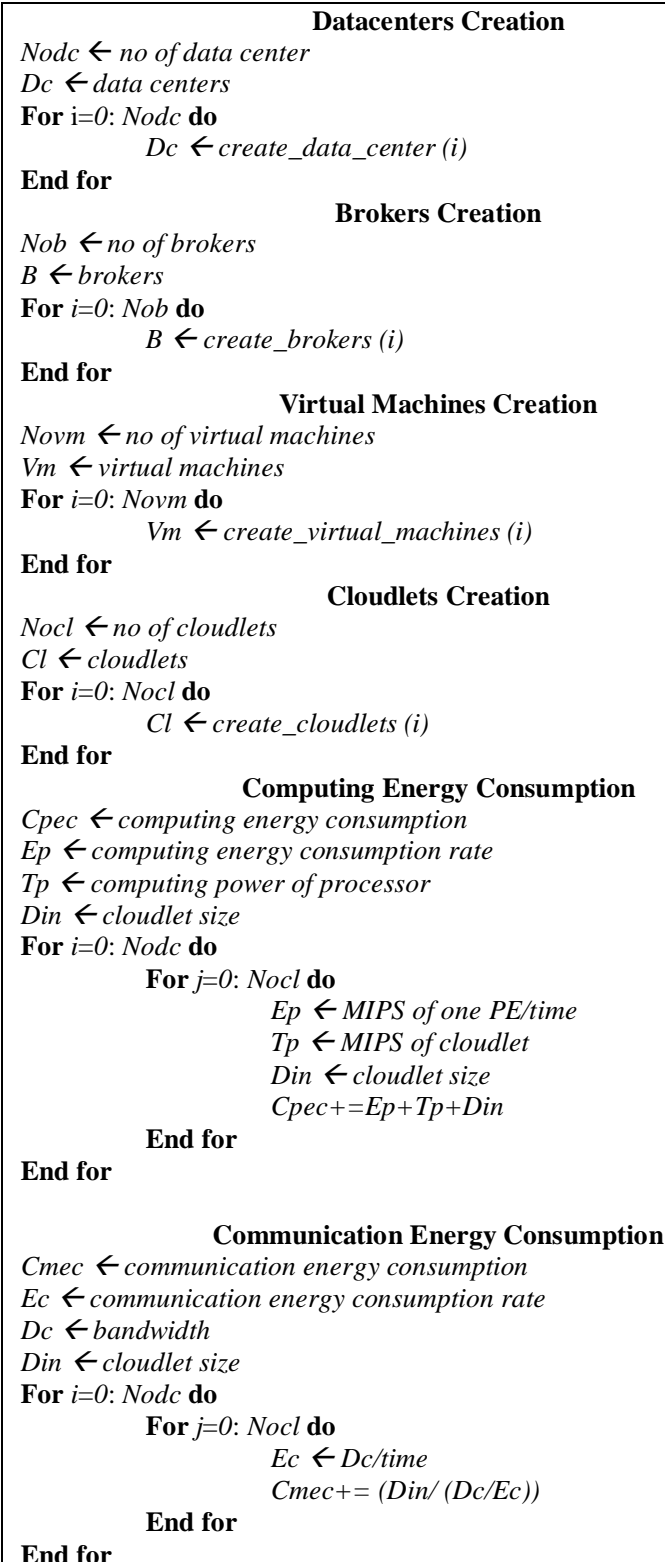

Enc $<$ energy consumption

For $i=0:$ Nodc do

End for

$$
\text { Enc }=\text { Cpec }(i)+C m e c(i)
$$

Th $\leftarrow$ threshold

Datacenters Clustering

To $\leftarrow$ total

For $i=0:$ Nodc do

End for

Th $\leftarrow$ To/Nodc

$C \leftarrow$ cluster

For $i=0:$ Nodc do

$$
\begin{aligned}
& \text { If Enc }(i)>\text { Th then } \\
& \text { Clse }
\end{aligned}
$$

\section{End if}

End for

$C \leftarrow$ cluster

Jobs Prioritization

For $i=0:$ Nocl do

If Nocl.Need $==$ "CPU" then

$C \leftarrow$ cluster 1

Else Nocl.Need $==$ "Memory" then

$C \leftarrow$ cluster 2

Else Nocl.Need $==$ "Bandwidth" then

$C \leftarrow$ cluster 3

End for

End if

Jobs Allocation

Alvm $\leftarrow$ allocated virtual machine

For $i=0$ : Nocl do

$$
\begin{aligned}
& \text { If } j==\text { Novm then } \\
& \text { End if }
\end{aligned}
$$

Alvm $=$ Virtual Machine $+j$

End for

$$
j++
$$

$\mathrm{Ou} \leftarrow$ over utilization

$U u \leftarrow$ under utilization

For $i=0$ : Nocl do

$$
\begin{gathered}
\text { If } \text { Nocl.Need }==\text { "CPU" then } \\
\text { If Nocl.Need }<=\text { Novm.i.capacity then } \\
U u \leftarrow \text { under utilization }
\end{gathered}
$$

Else

$$
\text { Ou } \leftarrow \text { over utilization }
$$

Else Nocl Need == "Memory" then

If Nocl.Need <= Novm.i.capacity then $U u \leftarrow$ under utilization

Else

$$
\text { Ou } \leftarrow \text { over utilization }
$$

Else Nocl.Need $==$ "Bandwidth" then

If Nocl.Need $<=$ Novm.i.capacity then $U u \leftarrow$ under utilization

Else

Ou $\leftarrow$ over utilization

End for

End if

$\mathrm{Dr} \leftarrow$ Dynamic reallocation

Dynamic Reallocation

For $i=0: O u$ do

$$
D r=\text { select } U u \text { Datacenter end for }
$$

\section{Performance Analysis}

The System executed using cloudsim tool having physical configuration of i3 processor, 4GB RAM and 1TB HDD.

Fig. 6 shows the energy consumption graph and it is observed that proposed methodology requires lower energy consumption. The $\mathrm{X}$-axis represents the number of VM whereas $\mathrm{Y}$-axis represents the total number of energy consumed measured in Kilowatt hour. As shown in graph for $100 \mathrm{VM}$ the total energy consumption by proposed methodology is $11.5 \mathrm{kwh}$, whereas AHDVC-MAD method consumes $14.9 \mathrm{Kwh}$.

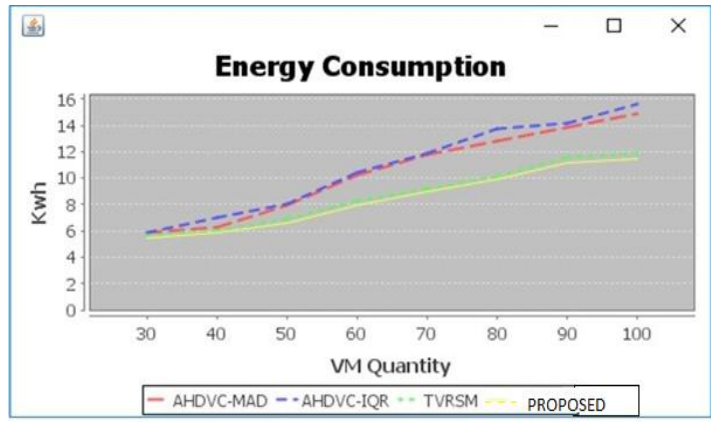

Fig.6: Graph for Energy Consumption 
Graph value of fig. 6 is summarized in to table 1 and it is observed that proposed methodology consumes energy as compared to all other method is very low.

\begin{tabular}{|l|l|l|l|l|}
\multicolumn{5}{c}{ Table 1: Energy Consumption Vs VM Quantity } \\
\hline \multirow{4}{*}{$\begin{array}{l}\text { AHDVC } \\
\text { VM } \\
\text { Quantity }\end{array}$} & $\begin{array}{l}\text { AHDVC- } \\
\text {-MAD } \\
\text { Energy } \\
\text { Con- } \\
\text { sumptio } \\
\text { n in } \\
\text { KWH }\end{array}$ & $\begin{array}{l}\text { Energy Con- } \\
\text { sumption in } \\
\text { KWH }\end{array}$ & $\begin{array}{l}\text { Energy Con- } \\
\text { sumption in } \\
\text { KWH }\end{array}$ & $\begin{array}{l}\text { Proposed } \\
\text { method- } \\
\text { Energy Con- } \\
\text { sumption in } \\
\text { KWH }\end{array}$ \\
\hline 30 & 5.9 & 5.9 & 5.7 & \\
\hline 40 & 6.3 & 7 & 6 & 5.5 \\
\hline 50 & 7.9 & 8 & 6.9 & 5.9 \\
\hline 60 & 10.2 & 10.4 & 8.3 & 6.6 \\
\hline 70 & 11.8 & 11.9 & 9.2 & 7.9 \\
\hline 80 & 12.8 & 13.8 & 10.1 & 9.9 \\
\hline 90 & 13.9 & 14.2 & 11.6 & 11.2 \\
\hline 100 & 14.9 & 15.6 & 11.9 & 11.5 \\
\hline
\end{tabular}

\section{Conclusion}

Minimizing the energy consumption is one of the critical stage to reduce the datacenter operating cost. Therefore, an innovative energy efficient resource management method is proposed to handle the resource scheduling and for the minimization of energy utilized by the cloud datacentres for the computational work. In proposed methodology once the energy consumption is calculated, threshold parameter is used to categorize the datacenter in to two clusters, cluster1 is for datacenters having energy consumption greater than threshold \& cluster2 is datacenters which are having energy consumption less than the threshold. Then cloudlets are allocated to different VM's of the different datacenters. In next step workload is predicted, if the workload is below zero means the datacenter is over utilized else datacenters are underutilized. VM's in the over utilized datacenters consumes more energy. Therefore, to save energy dynamic reallocation is performed to migrate VM's in the over utilized datacenters into underutilized datacenters, this can be done by considering the dynamic workload of the datacenters. The experimental results shown that the proposed technique outperforms than the all other techniques for higher resource utilization and to reducing the energy consumption.

\section{Acknowledgement}

FA would like to thanks KKWagh IEE\&R, Nashik for providing facilities to make successful work of this paper.

\section{References}

[1] M. Armbrustet al., "Above the clouds: A Berkeley view of cloud computing," University of California, Berkeley, Tech. Rep., Feb 2009 .

[2] Abhinandan S. Prasad, and Shrisha Rao,"A Mechanism Design Approach to Resource Procurement in Cloud Computing", IEEE Transactions on Computers, Vol. 63, No. 1, Pp.17-30, January 2014.

[3] Jens-Matthias Bohli, Nils Gruschka, Meiko Jensen, Member, IEEE ,Luigi Lo Iacono, and Ninja Marnau," Security and PrivacyEnhancing Multicloud Architectures" IEEE Transactions On Dependable \& Secure Computing, Vol. 10, No. 4, July/August 2013.

[4] En-Hao Chang, Chen-Chieh Wang, Chien-Te Liu, Kuan-Chung Chen, Student Member, Ieee, And Chung-Ho Chen, Member, IEEE," Virtualization Technology For Tcp/Ip Offload Engine", IEEE Transactions On Cloud Computing, Vol. 2, No. 2, April-June 2014
[5] Zhen Xiao, Senior Member, Ieee, Qi Chen, And Haipeng Luo," Automatic Scaling Of Internet Applications For Cloud Computing Services", IEEE Transactions On Computers, Vol. 63, No. 5, May 2014.

[6] Hui Zhang, Guofei Jiang, Kenji Yoshihira, And Haifeng Chen,'Proactive Workload Management In Hybrid Cloud Computing IEEE Transactions On Network And Service Management", Vol. 11, No. 1, March 2014

[7] S.K.Sonkar, .M.U.Kharat," A Review on Resource Allocation and VM Scheduling Techniques and a Model for Efficient Resource Management in Cloud Computing Environment", IEEE International conference on ICTBIG), ISBN: 978-1-5090-5519-9. Nov. 2016, DOI: 10.1109/ICTBIG.2016.7892646.

[8] A. Singh, M. Korupolu, and D. Mohapatra, "Server-storage virtualization: integration and load balancing in data centers," in Proc. of the ACM/IEEE conference on Supercomputing, 2008.

[9] Zhen Xiao, Senior Member, IEEE, Weijia Song, and Qi Chen, "Dynamic resource allocation using virtual machine in cloud computing environment," IEEE Transaction on Parallel and Distributed Systems, vol.24, no.6, pp.1107-1117, June 2013.

[10] W. E. Walsh, G. Tesauro, J. O. Kephart, and R. Das, "Utility Functions in Autonomic Systems," in ICAC '04: Proceedings of the First International Conference on Autonomic Computing. IEEE Computer Society, pp. 70-77, 2004.

[11] B. Kruekaew and W. Kimpan, "Virtual Machine Scheduling Management on Cloud Computing Using Artificial Bee Colony" Proceedings of the International MultiConference of Engineers and Computer Scientists 2014 Vol I, IMECS 2014, March 12 - 14, 2014, Hong Kong.

[12] J. Hu, J. GU, G. Sun, and T. Zhao, "A scheduling strategy on load balancing of virtual machine resources in cloud computing environment," in 3rd IEEE Int. Symp. On Parallel Architectures, Algorithms and Programming (PAAP), 2010, 18-20 Dec. 2010, pp.89-96.

[13] G. Raj, "Effective Cost Mechanism for Cloudlet Retransmission and Prioritized VM Scheduling Mechanism Over Broker Virtual Machine Communication Framework", International Journal on Cloud Computing: Services and Architecture, pp.41-50, 2012

[14] Bo Li; Jianxin Li, Jinpeng Huai, Tianyu Wo; Qin Li; Liang Zhong,"EnaCloud: An Energy-Saving Application Live Placement Approach for Cloud Computing Environments".IEEE International Conference on Cloud Computing, Pages: 17-24, Year: 2009, DOI: 10.1109/CLOUD.2009.72

[15] Xin Li; Zhuzhong Qian; Ruiqing Chi; Bolei Zhang; Sanglu Lu," Balancing Resource Utilization for Continuous Virtual machine Requests in Clouds", Sixth International Conference on Innovative Mobile and Internet Services in Ubiquitous Computing (IMIS),IEEE Conference Publication 2012 ,Pages: 266273, DOI: 10.1109/IMIS.2012.72

[16] AtonBelglazov,RajkumarBuyya,'Energy Efficient Allocation of Vi rtual Machines in Cloud Data Centers",10th IEEE international Conference on Cluster, Cloud and Grid Computing (CCGrid), 2010 , Pages: 577 - 578,Year:2010, DOI: 10.1109/CCGRID.2010. 45

[17] Mehiar Dabbagh, Bechir Hamdaoui, Mohsen Guizaniy and Ammar Rayesz "An Energy-Efficient VM Prediction and Migration Framework for Overcommitted Clouds", IEEE Transaction on Cloud Computing DOI 10.1109/TCC.2016.2564403

[18] Fei Taoa, Ying Fengb, Lin Zhanga, and T.W. Liaoc "CLPS-GA: A case library and Pareto solution-based hybrid genetic algorithm for energy-aware cloud service scheduling "Science direct-Applied Soft Computing, vol. 19, june2014, PP.264-279.

[19] Tarandeep Kaur, Inderveer Chana "Energy aware scheduling of deadline constrained tasks in cloud Computing", 4 April 2016 (C) Springer cluster computing 2016.

[20] Jieun Choi, Theodora Adufu, Yoonhee Kim, Seoyoung Kim, Soonwook Hwang “A Job Dispatch Optimization Method on Cluster and Cloud for Large-scale High-Throughput Computing Service", IEEE international conference on cloud and Automatic Computing Catalogue number 978-1-4673-3/15, DOI : 10.1109/ICCAC.2015.42

[21] A.V.Karthick, .E.Ramara, R.Ganapathy Subramanian, "An Efficient Multi Queue Job Scheduling for Cloud Computing”, IEEE World Congress on Computing and communication Technologies, catalogue number 978-1-4799-28774/14,DOI:10.1109/WCCCT.2014.8

[22] Vahid Arabnejad, Kris Bubendorfer, Bryan Ng and Kyle Chard "A Deadline Constrained Critical Path Heuristic for Costeffectively Scheduling Workflows", 2015 IEEE/ACM $8^{\text {th }}$ Interna- 
tional conference on utility and cloud computing, catalogue number:978-0-7695-5697-0/15, DOI: 10.1109/UCC.2015.4

[23] Zhiming Wang, Kai Shuang, Long Yang, Fangchun Yang "Energy-aware and revenue enhancing Combinatorial Scheduling in Virtualized of Cloud Datacenter", Journal of Convergence Information Technology(JCIT)Volume7, Number1, January 2012 ,DOI: $10.4156 /$ jcit.

[24] Daochao Huang, Peng Du, Chunge Zhu, Hong Zhang, Xinran Liu“Multi-resource Packing for Job Scheduling in Virtual Machine Based Cloud Environment", IEEE symposium on Service Oriented System Engineering, Catalogue number: 978-1-4799-8365, DOI:

10.1109/SOSE.2015.30.

[25] Ryan Jansen, Paul R. Brenner, "Energy Efficient Virtual Machine Allocation in the Cloud an Analysis of Cloud Allocation Policies", $2^{\text {nd }}$ IEEE conference on Green computing, Catalogue number: 978$1-4577-1221-0 / 11$

[26] Shahin Vakilinia, Behdad Heidarpour and Mohamed Cheriet, Energy "Efficient Resource Allocation in Cloud Computing Environments", IEEE special section on Future network: Arch. Protocols and Applications vol.4, 2016, PP.8544-8557. 